\title{
Small marker chromosomes in man: origin from pericentric heterochromatin of chromosomes 1, 9, and 16
}

\author{
D F Callen, M L Ringenbergs, J C S Fowler, C J Freemantle, E A Haan
}

\begin{abstract}
Three patients with different marker chromosomes were screened by in situ hybridisation using biotinylated probes to chromosome specific pericentric repeats to determine the chromosomal origin of the marker. Each marker had a different origin, with one from each of chromosomes 1, 9, and 16. This is the first time that autosomal marker chromosomes consisting of a small ring have been shown to be derived from the pericentric heterochromatin of metacentric and submetacentric chromosomes. Evidence suggests that such markers are not associated with any significant risk of phenotypic abnormalities, but additional cases need to be studied.
\end{abstract}

Marker chromosomes occur in humans with a frequency of approximately 1.5 per 1000 with $40 \%$ being familial.' These markers vary in both size and structure. A de novo marker that is small, has an absence of obvious euchromatin, and has distamycin/ DAPI positive material seems to be associated with a low risk of fetal anomalies.' However, studies using conventional cytogenetic staining procedures generally cannot identify the chromosomal origin of marker chromosomes. Among the smaller marker chromosomes an exception is the inv $\operatorname{dup}(15)$ where the presence of distamycin/DAPI positive and NOR staining material confirms the origin. ${ }^{23}$

Cytogenetics Unit, Adelaide Children's Hospital, North Adelaide, South Australia 5006.

D F Callen, $M$ L Ringenbergs

School of Biological Sciences, Flinders University of South Australia, Bedford Park, South Australia.

J C S Fowler

Department of Medical Genetics, Adelaide Children's Hospital, North Adelaide, South Australia.

C J Freemantle, E A Haan

Correspondence to Dr Callen.

Received for publication 4 September 1989.

Accepted for publication 14 September 1989.
DNA probes derived from the pericentric repeat sequences, either of satellite II, satellite III, or from alphoid repeats, can be chromosome specific and thus provide a means to determine the chromosomal origin of marker chromosomes. ${ }^{+5}$ This report presents the identification by in situ hybridisation with biotinylated pericentromeric repeat probes of three different small marker chromosomes ascertained in patients during routine cytogenetic investigations.

\section{Materials and methods}

PROBES

All the probes used were derived from pericentric repeats which were classified as either alphoid (probes p308, ${ }^{6}$ RR38, ${ }^{7}$ Po10RP8, ${ }^{8}$ L $1.26,{ }^{9}$ TR17, ${ }^{10}$ and L1.84 $4^{9}$ ), satellite II (probes RR6 ${ }^{7}$ and pHuR $195^{5}$ ), or satellite III (probe $228 S^{\prime \prime}$ ) (table).

\section{IN SITU HYBRIDISATION}

Each probe was labelled with biotin-11-dUTP or biotin-7-dATP using a nick translation kit (BRL, USA). Metaphase spreads were prepared from short term PHA stimulated lymphocyte cultures using standard methods. Chromosomal DNA was denatured in $70 \%$ deionised formamide at $70^{\circ} \mathrm{C}$ for two minutes followed by dehydration in an ice cold alcohol series.

Results of in situ hvbridisation of biotinvlated specific centric repeat probes with high stringency wash conditions to metaphase chromosomes from three patients with marker chromosomes.

\begin{tabular}{lcccc}
\hline & $\begin{array}{c}\text { Chromosome } \\
\text { detected }\end{array}$ & 1 & 2 & 3 \\
\cline { 3 - 5 } Probe & 1 & + & - & - \\
\hline RR6 & 6 & - & - & - \\
p308 & 7 & - & - & - \\
RR38 & 9 & - & + & - \\
228S & 10 & - & - & - \\
pu10RP8 & 13,21 & - & - & + \\
L1.26 & 16 & - & - & - \\
pHuR195 & 17 & - & - & - \\
TR17 & 18 & & \\
L1.84 & 18 & &
\end{tabular}

+indicates that there was label on the marker chromosome. 
Probes were diluted at optimal concentrations in hybridisation buffer $(2 \times \mathrm{SSC}(300 \mathrm{mmol} / \mathrm{l} \mathrm{NaCl}, 30$ $\mathrm{mmol} / \mathrm{l} \mathrm{NaCitrate}), 50 \%(\mathrm{v} / \mathrm{v})$ deionised formamide, $5 \%(\mathrm{w} / \mathrm{v})$ dextran sulphate, $0.1 \mathrm{mmol} / 1$ disodium EDTA, $0.05 \mathrm{mmol} / 1 \mathrm{Tris} \mathrm{HCl}, \mathrm{pH} 7 \cdot 3$, and $50 \mu \mathrm{g} / \mathrm{ml}$ sheared salmon sperm DNA). After denaturation by heating to $100^{\circ} \mathrm{C}$ for five minutes and snap chilling on ice for five minutes, $25 \mu \mathrm{l}$ of the probe was pipetted onto the slide, coverslipped, and sealed with rubber cement. Hybridisation was carried out at $37^{\circ} \mathrm{C}$ in a humidified chamber for 17 hours. After hybridisation, slides were washed in $2 \times$ SSC for 30 minutes at $25^{\circ} \mathrm{C}, 0.1 \times S S C$ for 30 minutes at $65^{\circ} \mathrm{C}, 2$ $\times$ SSC for 15 minutes at $25^{\circ} \mathrm{C}$, and finally in PBT $(0.001 \mathrm{~mol} / \mathrm{l}$ phosphate buffered saline, $\mathrm{pH} 7 \cdot 4$ (PBS), $3 \%$ BSA (Sigma), and $0 \cdot 1 \%$ Triton $\mathrm{X}-100$ ) for 15 minutes at $25^{\circ} \mathrm{C}$.

The hybridised probe was detected by first incubating with rabbit anti-biotin IgG (Enzo, USA) for 60 minutes at $37^{\circ} \mathrm{C}$. Slides were washed $3 \times$ five minutes in PBS-Triton X (PBS and $0.1 \%$ Triton $\mathrm{X}-100$ ) followed by further incubation with peroxidase conjugated sheep anti-rabbit Ig (Silenus, Australia) for 60 minutes at $37^{\circ} \mathrm{C}$. After washing $3 \times$ five minutes in PBS-Triton $\mathrm{X}$ and five minutes in PBS, the slides were incubated with $0.5 \mathrm{mg} / \mathrm{ml} \mathrm{DAB}\left(3,3^{\prime}\right.$ diaminobenzidine tetrahydrochloride) (BRL, USA) in PBS containing $0.012 \%(\mathrm{v} / \mathrm{v})$ hydrogen peroxide for five minutes. Slides were washed in distilled water for five minutes and air dried. The final amplification of the $\mathrm{DAB} /$ hydrogen peroxide signal was by silver deposition using the procedure as detailed by Burns $e t$ $a l .{ }^{12}$

Banded metaphase preparations were used initially to determine the localisation of probe signal. Specific hybridisation for each probe could be assessed by a clear signal on the expected normal pair of homologues (or in the case of probe L1.26 on two pairs of homologues, namely chromosomes 13 and 21) with an absence of signal on other chromosomes. The marker was considered negative if there was an absence of signal in 10 such metaphases.

\section{Case reports}

Patient 1 was a girl born at term weighing $2600 \mathrm{~g}$ after a normal pregnancy, labour, and delivery. She was reviewed at 13 years of age. Clinical features included microcephaly (head circumference $46.5 \mathrm{~cm}$ ), short stature (height $141.5 \mathrm{~cm}$ ), moderately severe mental retardation, myopia, and dysmorphic facial features comprising a low anterior hairline, synophrys, short palpebral fissures, narrow nose, high nasal bridge, short philtrum, thin upper lip, and anomalies of ear folding. In the past there had been congenital nystagmus, bilateral vesicoureteric reflux, and chronic serous otitis media. $X$ rays showed spina bifida occulta of $\mathrm{S} 1$.
Patient 2 was a girl diagnosed as having a marker chromosome after amniocentesis was performed on her 38 year old mother. Her parents were counselled about the possibility of clinical effects in their daughter as a result of the extra chromosome, but decided to continue the pregnancy. The mother developed unilateral hydronephrosis during the pregnancy as a result of pelviureteric obstruction, which was treated surgically.

The child was born after a normal labour and delivery with Apgar scores of 8 at one minute and 9 at five minutes. Birth weight was $3200 \mathrm{~g}$, length was $49 \mathrm{~cm}$, and head circumference was $32.5 \mathrm{~cm}$. Gastrooesophageal reflux needed treatment until 8 months of age. Subsequently, general health has been good. When reviewed at $4 \frac{1}{2}$ years, her growth and development were normal and she had no dysmorphic features.

Patient 3 was a male born at term, birth weight $3325 \mathrm{~g}$, after a normal pregnancy, labour, and delivery. Significant resuscitation was needed at birth. The first week of life was marked by vomiting and mild jaundice but subsequently he fed satisfactorily and thrived, although being described as irritable for 12 months. There was concern about his development from 3 months of age.

At 33 years of age, the clinical features included microcephaly (head circumference $52.3 \mathrm{~cm}$ ), severe mental retardation, severe spastic quadriparesis, scoliosis, pectus excavatum, and dysmorphic facial features comprising a long face with a long nose and prominent chin, smooth philtrum, thin upper lip, and strabismus.

\section{Results}

The karyotype of patient 1 is $46, \mathrm{XX}, \operatorname{del}(18)(\mathrm{q} 22) /$ $47, \mathrm{XX}$,del(18)(q22), +mar (fig 1). The proportion of cells with the marker was $25 \%$. The mental retardation and malformations are consistent with the presence of a del(18)(q22). Parental chromosome studies were normal.

The karyotype of patient 2 is $46, \mathrm{XX} / 47, \mathrm{XX},+$ mar (fig 1). This marker was present in $40 \%$ of cultured amniocytes. After birth, short term lymphocyte cultures showed the marker in a similar proportion of cells. Parental chromosome studies were normal.

The karyotype of patient 3 is $46, \mathrm{XY} / 47, \mathrm{XY},+$ mar (fig 1). Approximately $75 \%$ of cells scored possessed the marker chromosome. Family studies showed the marker chromosome was present in short term lymphocyte cultures in both the mother and sister in $73 \%$ and $37 \%$ of cells, respectively. Both the mother and sister were normal.

The appearance of each marker was similar (fig 1), being approximately the size of the short arm of chromosome 18 with an appearance suggestive of a ring. The marker of patient 1 was slightly larger than 


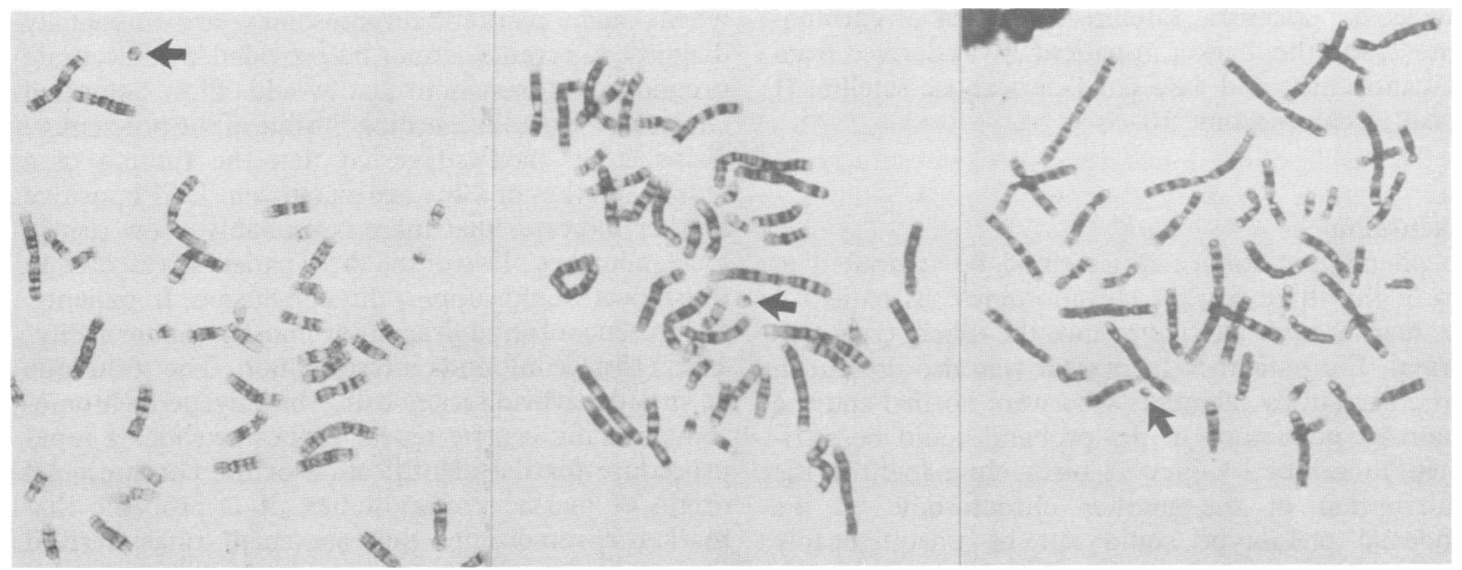

Figure 1 G banded metaphase or partial metaphase showing marker chromosome (arrowed). Left: patient 1. Centre: patient 2. Right: patient 3.

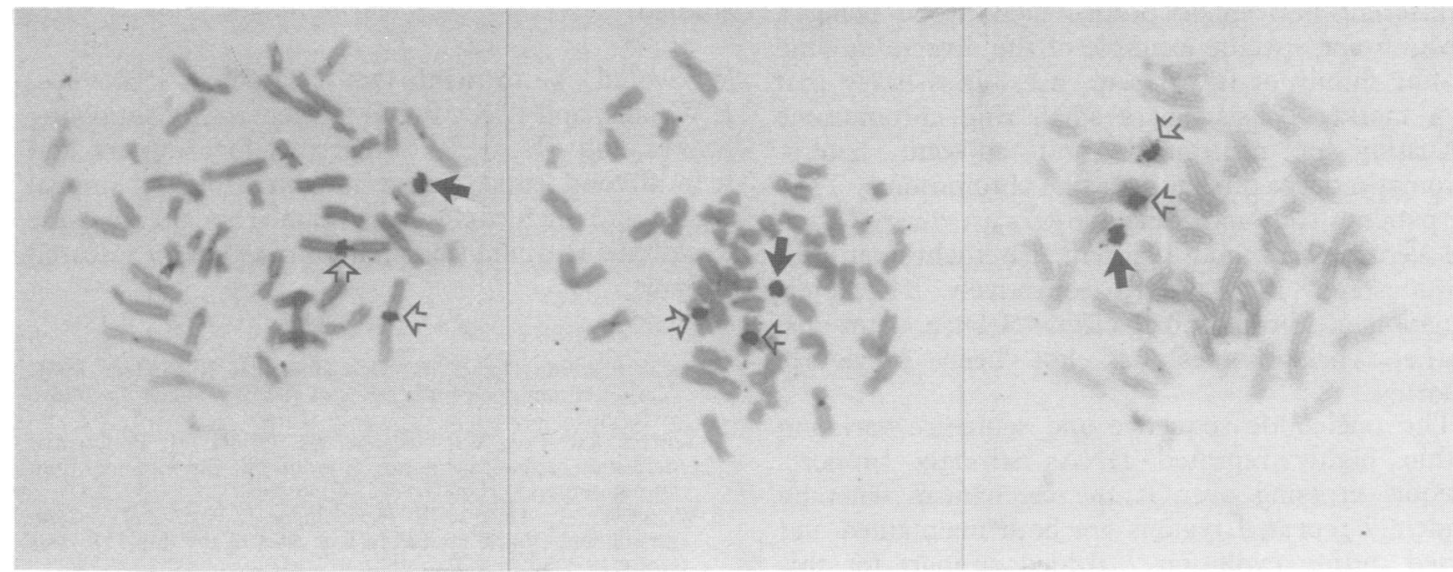

Figure 2 In situ hybridisation of biotinylated pericentric repeat probes showing signal on marker chromosome. The signal of the marker chromosome is indicated by the solid arrow and the signal on the two normal homologues is indicated by the open arrow. Left: patient 1 , probe RR6 specific for chromosome 1 .

Centre: patient 2, probe $228 S$ specific for chromosome 9.

Right: patient 3, probe pHuR195 specific for chromosome 16.

in the other two patients. All three markers were C band positive and showed bright blue fluorescence with distamycin/DAPI banding. This would suggest that they are predominantly of heterochromatin, which could have originated from chromosomes 1,9 , 15,16 , or $\mathrm{Y}$.

For each biotinylated probe, preliminary in situ hybridisation experiments established the optimum concentration of probe in the hybridisation mix, which allowed development of a clear signal on the target chromosomes with negligible background signal on the remainder of the chromosome complement. For each probe, high stringency of wash conditions restricted the hybridisation signal to a single chromosome, except for probe L1.26 which detected signal on chromosomes 13 and 21 (table). The chromosomes detected by each probe were the same as previously described. ${ }^{5-10}$

The results of in situ hybridisation are presented in the table and fig 2. For patient 1 , in situ hybridisation studies showed that, of the probes used, only RR6 was positive. This showed that the marker chromosome in this patient contained the satellite II repeat of chromosome 1 and therefore was presumably derived from the centromere and a portion of the pericentric satellite II repeat DNA of this chromosome. Similarly, it was determined that the marker in patient 2 was derived from the centromere and 
associated pericentric satellite III repeat of chromosome 9, and the marker in patient 3 was derived from the centromere and associated pericentric satellite II repeat of chromosome 16 .

\section{Discussion}

No phenotypic abnormalities could be attributed to two of the three marker chromosomes. In patient 2 the marker was de novo and the phenotype was normal. For patient 3 the marker was also present in two other family members who were normal and the abnormal phenotype in the proband could be attributed to cerebral injury at birth. In patient 1 the contribution of the marker chromosome to the abnormal phenotype could not be unequivocably ascertained because a deletion was also present. The clinical phenotype was consistent with the presence of the $\operatorname{del}(18)(\mathrm{q} 22) .{ }^{13}$

Each of the marker chromosomes was positive with a different pericentric probe, each probe being a chromosome specific example of the diverse satellite III-like family of repeat sequences. It is likely that each marker consists of a small ring chromosome consisting of centromere and adjacent heterochromatin derived from the same chromosome. Thus for patients 1, 2, and 3 the markers are derived from the centromeres and adjacent heterochromatin of chromosomes 1,9 , and 16 respectively. The determination of chromosomal origin of such autosomal marker chromosomes has not been previously reported.

The nucleotide sequence and sequence variation within highly repeated DNAs strongly supports unequal crossing over as the mechanism whereby tandemly repeated regions are both maintained and varied during evolution. ${ }^{1+}$ Added support for this concept comes from studies of variation in human ribosomal DNA. ${ }^{15}$ However, while unequal crossing over can generate the interchromosomal variation in centric heterochromatin that is commonly observed, it cannot classically generate an intrachromosomal rearrangement, such as the ring chromosomes described in this report. To generate these ring chromosomes a possible mechanism is two ' $U$ ' type chromatid exchanges that involve the same pair of chromatids and are on opposite sides of the centromere. Alternatively, breakage of the same chromatid on either side of the centromere and subsequent reunion of the ends adjacent to the centromere would also generate a ring chromosome. Breakage and reunion events involving the same chromatid have also been suggested for the origin of an unusual apparent $\mathrm{i}(\mathrm{Xq})$ chromosome that contains a small segment of $\mathrm{Xp} .^{16}$ This may be a significant mechanism for the generation of pericentric rearrangements.

The risk for phenotypic abnormalities caused by a marker chromosome is generally unclear. Therefore, when such marker chromosomes are prenatally diagnosed, parents cannot be provided with accurate prognostic information that would allow informed choices to be made regarding the fate of the pregnancy. Sachs $e t a l^{1}$ have suggested that the finding of a familial marker or a de novo distamycin/DAPI positive marker indicates that there is probably a low risk of fetal anomalies. Two of the three patients presented in this report would support this conclusion. In patient 1 the presence of an additional chromosome abnormality, a del (18q), confounds interpretation. The technique of in situ hybridisation using biotinylated chromosome specific centric repeat probes provides a rapid procedure for the identification of the chromosomal origin of marker chromosomes. It is probable that marker chromosomes that are small rings derived from the centromere and heterochromatin of chromosomes 1,9 , and 16 do not have any increased risk of phenotypic abnormalities. However, to confirm this, additional patients will need to be studied.

We would like to thank Drs K H Choo, P Devilee, $\mathrm{E} \mathrm{W}$ Jabs, and R K Moyzis for generously supplying probes and Dr G $R$ Sutherland for support and helpful comments. We are indebted to the National Health and Medical Research Council and the Adelaide Children's Hospital Research Foundation for research support.

1 Sachs ES, Van Hemel JO, Den Hollander JC, Jahoda MGJ. Marker chromosomes in a series of 10,000 prenatal diagnoses. Cytogenetic and follow-up studies. Prenat Diagn 1987;7:81-9.

2 Schreck RR, Breg WR, Erlanger BF, Miller OJ. Preferential derivation of abnormal human G-group-like chromosomes from chromosome 15. Hum Genet 1977;36:1-12.

3 Wisniewski L, Hassold T, Heffelfinger J, Higgins JV. Cytogenetic and clinical studies in five cases of inv dup (15). Hum Genet 1979;50:259-70.

4 Willard HF, Waye JS. Hierarchical order in chromosome-specific human alpha satellite DNA. Trends Genet 1987;3:192-8.

5 Moyzis RK, Albright KL, Bartholdi MF, et al. Human chromosome-specific repetitive DNA sequences: novel markers for genetic analysis. Chromosoma 1987;95:375-86.

6 Jabs EW, Persico MG. Characterization of human centromeric regions of specific chromosomes by means of alphoid DNA sequences. Am 7 Hum Genet 1987;41:374-90.

7 Fowler JCS, Skinner JD, Burgoyne LA, Ringenbergs ML, Callen DF. Restriction enzyme enrichment of chromosome-specific tandem repetitive sequences: a $9 \mathrm{bp}$ sequence common to satellite alphoid and satellite II is a possible protein binding site (submitted).

8 Devilee P, Kievits T, Waye JS, Pearson PL, Willard HF. Chromosome-specific alpha satellite DNA: isolation and mapping of a polymorphic alphoid repeat from human chromosome 10. Genomics 1988;3:1-7.

9 Devilee P, Cremer T, Slagboom PE, et al. Two subsets of human alphoid repetitive DNA show distinct preferential localization in the pericentric regions of chromosomes 13,18 and 21 . Cytogenet Cell Genet 1986;41:193-201.

10 Choo KH, Brown R, Webb G, Craig IW, Filby RG. Genomic organisation of human centromeric alpha satellite DNA: characterization of a chromosome 17 alpha satellite sequence. DNA 1987;6:297-305.

11 Fowler JCS, Burgoyne LA, Baker EG, Ringenbergs ML, Callen DF. Human satelite III DNA: genomic location and sequence homogeneity of the Taql-deficient polvmorphic sequences. Chromosoma (in press).

12 Burns J, Chan VTW, Jonasson JA, Fleming KA, Taylor S, 
McGee JO'D. Sensitive system for visualising biotinylated DNA probes hybridised in situ: rapid sex determination of intact cells. I Clin Pathol 1985;38:1085-92.

13 Schinzel A. Catalogue of unbalanced chromosome aberrations in man. Berlin: de Gruyter, 1984.

14 Brutlag DL. Molecular arrangement and evolution of heterochromatic DNA. Annu Rev Genet 1980;14:121-44.
15 Erickson JM, Schmickel RD. A molecular basis for discrete size variation in human ribosomal DNA. Am $\mathcal{f}$ Hum Genet 1985;37:311-25.

16 Callen DF, Mulley JC, Baker EG, Sutherland GR. Determining the origin of human $X$ isochromosomes by use of DNA sequence polymorphisms and detection of an apparent $\mathbf{i}(\mathbf{X q})$ with Xp sequences. Hum Genet 1987;77:236-40. 